\title{
Gradhiva
}

GRADHIV

Revue d'anthropologie et d'histoire des arts

\section{Discours prononcé par Aimé Césaire à Dakar le} 6 avril 1966

dans le cadre du Colloque sur l'art dans la vie du peuple qui marqua l'ouverture du Premier Festival mondial des arts nègres (30 mars 21 avril 1966)

\section{(2) OpenEdition}

Journals

Édition électronique

URL : http://journals.openedition.org/gradhiva/1604

DOI : 10.4000/gradhiva. 1604

ISSN : 1760-849X

Éditeur

Musée du quai Branly Jacques Chirac

Édition imprimée

Date de publication : 4 novembre 2009

Pagination : 208-215

ISBN : 978-2-35744-012-8

ISSN : 0764-8928

Référence électronique

" Discours prononcé par Aimé Césaire à Dakar le 6 avril 1966 », Gradhiva [En ligne], 10 | 2009, mis en ligne le 05 février 2010, consulté le 10 décembre 2020. URL : http://journals.openedition.org/gradhiva/ 1604 ; DOI : https://doi.org/10.4000/gradhiva.1604 


\title{
Discours prononcé par Aimé Césaire à Dakar le 6 avril 1966
}

\author{
dans le cadre du Colloque sur l'art dans la vie du peuple \\ qui marqua l'ouverture du Premier Festival mondial des \\ arts nègres (30 mars - 21 avril 1966)
}

Monsieur le Président,
Mesdames, Messieurs,

Je voudrais d'abord vous faire part des hésitations que j'ai eues à prendre la parole dans ce colloque. Je ne suis à aucun degré un homme de science, à aucun degré un expert, et j'ai conscience que dans une telle assemblée, j'ai beaucoup plus à apprendre qu'à enseigner.

Aussi bien vous demanderai-je de ne voir dans cette intervention que la manifestation du désir que j'ai à vous dire à quel point j'ai été intéressé par vos travaux et de vous soumettre à titre de contribution quelques réflexions qui sont nées de mon expérience d'homme de culture, de mon expérience de poète, de mon expérience d'homme antillais à propos de l'Afrique mère.

Le thème de ce colloque est ainsi formulé : «Fonction et signification de l'art négro-africain dans la vie du peuple et par le peuple». Je crois que pour répondre à cette question particulière le plus simple est de poser d'abord une question plus générale et de tâcher d'y répondre et cette question serait celle-ci : «Fonction et signification de l'art dans le monde moderne ». Autrement dit, avant de parler de l'art africain et de sa signification pour l'Afrique moderne, le mieux m'apparait de parler de l'art tout court et de sa fonction dans le monde tout court.

Pourquoi, dans le monde comme il va, il est apparu essentiel aux organisateurs de ce colloque, pourquoi il nous apparait essentiel à nous autres, hommes de culture, de valoriser la fonction de l'art? Car enfin l'art n'est pas toute la culture, il n'en est qu'un aspect. Alors pourquoi privilégier cet aspect au détriment des autres aspects de la culture? Je répondrai que c'est un signe des temps et que si nous avons, d'un propos délibéré, choisi de privilégier l'art, c'est que nous estimons que jamais comme aujourd'hui le monde n'a eu autant besoin de l'art.

Ou'on le veuille ou non, il y a à l'heure actuelle une civilisation éminente et tentaculaire. C'est la civilisation européo-américaine, la civilisation industrielle qui couvre le monde de son réseau, et atteint désormais - car il est clair que maintenant, nous sommes entrés dans l'ère du monde fini - les points les plus reculés du globe. Il est inutile de rappeler les mérites de cette civilisation européenne. Ils sont nombreux et éclatants. Mais, pour comprendre le rôle de l'art, notre besoin d'art et de poésie, c'est plutôt son côté négatif qu'il faut rappeler.

L'homme de la civilisation européenne est un homme qui a mis au point un système de pensée qui lui a permis de vaincre et de dominer la nature. Mais il est arrivé à notre conquérant une singulière mésaventure : il a fini par être vaincu par sa propre puissance : il est devenu le prisonnier et la victime des concepts et des catégories qu'il avait inventés pour appréhender le monde. Mieux, avec la pensée européenne moderne (je dis bien moderne car l'Europe n'a pas toujours été ce qu'elle est) est né un processus nouveau, celui que certains penseurs ont appelé un processus de réification, c'est-à-dire de chosification du monde.

De quoi s'agit-il? Il s'agit de la substitution à la totalité dialectique qu'est le monde, de la substitution au monde concret et hétérogène, donc riche et varié, d'une véritable algèbre 
d'abstractions homogénéisées et dissociées représentant un abrégé du monde, commode, sans doute, mais correspondant à un appauvrissement et à un succédané du monde. Les conséquences, vous les connaissez, c'est l'apparition du monde mécanisé, du monde de l'efficience, mais aussi du monde où l'homme devient chose lui-même, du monde où le temps n'est plus le temps, mais une manière d'espace, rempli de choses quantitativement mesurables. Bref, nous sommes en face d'une dévalorisation progressive du monde, qui débouche très naturellement sur l'apparition d'un univers inhumain sur la trajectoire duquel se trouvent le mépris, la guerre, l'exploitation de l'homme par l'homme. C'est cela, cette invasion du monde et de l'homme par les choses, c'est ce processus de réification du monde installé par la culture européenne dans la société, qui explique que le besoin d'art et de poésie soit aujourd'hui un besoin véritablement vital, dans le sens où on dit que l'art est vital pour l'homme.

Comme l'homme a besoin d'oxygène pour survivre, il a besoin d'art et de poésie. Il sait, en effet, au contraire de la pensée conceptuelle, au contraire de l'idéologie, que l'art et la poésie rétablissent la dialectique de l'homme et du monde. Par l'art, le monde réifié redevient le monde humain, le monde des réalités vivantes, le monde de la communication et de la participation. D'une collection de choses, la poésie est jeunesse. Elle est cette force qui redonne au monde sa vitalité première, qui redonne à chaque chose son aura de merveilleux en la replaçant dans la totalité originelle. Si bien que sauver la poésie, sauver l'art, c'est en définitive sauver l'homme moderne en personnalisant et en revitalisant la nature.

Si on avait besoin d'une preuve, je dirais qu'il n'est que de constater que jamais le besoin poétique ne se fait autant sentir, que jamais l'homme ne s'accorde, ne s'accroche à la poésie de manière si désespérée, comme à une dernière planche de salut, qu'au sortir de ces époques pleines de bruit et de fureur qui s'appellent la guerre, que cette guerre soit chaude ou froide, précisément au sortir de ces époques où la non-communication et la chosification se sont exacerbées à un degré proprement intolérable. Et, ici, je pense au surréalisme européen qui a suivi la Première Guerre mondiale, et, puisque je parle au nom des poètes d'Afrique et des poètes d'Afrique francophone, permettez-moi d'évoquer ici l'ensemble du mouvement poétique dit de la négritude.

Mes chers amis, je dois vous dire tout de suite qu'aucun mot ne m'irrite davantage que le mot «négritude » - je n'aime pas du tout ce mot-là, mais puisqu'on l'a employé et puisqu'on l'a tellement attaqué, je crois vraiment que ce serait manquer de courage que d'avoir l'air d'abandonner cette notion. Je n'aime pas du tout le mot «négritude » et je dois vous dire que cela m'irrite toujours lorsque, dans les conférences internationales où il y a des anglophones et francophones, on introduit cette notion qui m'apparaît comme une notion de division. La négritude est ce qu'elle est, elle a ses qualités, elle a ses défauts, mais au moment où on la vilipende, où on la dénature, je voudrais quand même que l'on fasse réflexion sur ce qu'était la situation des Nègres, la situation du monde nègre, au moment où cette notion est née, comme spontanément, tellement elle répondait à un besoin. Bien sûr, à l'heure actuelle, les jeunes peuvent faire autre chose, mais, croyez-moi, ils ne pourraient pas faire autre chose à l'heure actuelle si, à un certain moment, entre 1930 et 1940, il n'y avait pas eu des hommes qui avaient pris le risque de mettre sur pied ce mouvement dit de la négritude. Ce mouvement de la négritude tellement attaqué, et tellement défiguré, il ne faut pas oublier le rôle qu'il a joué dans l'éveil du monde nègre, dans l'éveil de l'Afrique. Quand je lis une phrase comme celle que Saint-John Perse a prononcée lorsqu'il a reçu le prix Nobel, quand il a écrit ceci : «Quand la mythologie s'effondre, c'est dans la poésie que trouve refuge le divin. Peutêtre même son relais et jusque dans l'ordre social et l'immédiat humain, quand la porteuse de pain de l'antique cortège cède son pain aux porteuses de flambeaux, c'est à l'imagination poétique que s'allume encore la haute passion des peuples en quête de clarté. » Si la négritude a bien mérité de l'Afrique, c'est que précisément, dans l'étendue de l'abomination et de la nuit, ses poètes ont été, malgré leurs défauts, des porteurs de clarté. 
Cette notion de la négritude, on s'est demandé si ce n'était pas un racisme. Je crois que les textes sont là. Il suffit de les lire et n'importe quel lecteur de bonne foi s'apercevra que, si la négritude est un enracinement particulier, la négritude est également dépassement et épanouissement dans l'universel.

Pour en revenir à mon propos, je dirai, à propos de la négritude, que, dans la perspective de la réification, le racisme et le colonialisme avaient tenu à transformer le Nègre en chose. L'homme noir n'était plus appréhendé par l'homme blanc qu'à travers le prix d'une déformation, de stéréotypes, car c'est toujours de stéréotypes que vivent les préjugés. Et c'est cela le racisme. Le racisme, c'est la non-communication. C'est la chosification de l'autre, du Nègre ou du Juif; la substitution à l'autre de la caricature de l'autre, une caricature à laquelle on donne valeur d'absolu. L'apparition de la littérature de la négritude et de la poésie de la négritude n'a produit un tel choc que parce qu'elles ont dérangé l'image que l'homme blanc se faisait de l'homme noir, qu'elles ont marqué avec ses qualités, avec ses défauts, donc avec sa charge d'homme, dans le monde des abstractions et des stéréotypes que l'homme blanc s'était jusque-là fabriqué à son sujet de manière unilatérale.

Et c'est bien cela, je crois, le service que la négritude a rendu au monde. C'était par là contribuer à l'édification d'un véritable humanisme, de l'humanisme universel, car enfin il n'y a pas d'humanisme s'il n'est pas universel, et il n'y a pas d'humanisme sans dialogue, et il ne peut y avoir de dialogue entre un homme et une caricature.

En restituant l'homme noir dans sa stature humaine, dans sa dimension humaine, pour la première fois, la littérature de la négritude a rétabli les possibilités de dialogue entre l'homme blanc et l'homme noir et ce n'est pas un de ses moindres mérites. Il est très vrai que la littérature de la négritude a été une littérature de combat, une littérature de choc et c'est là son honneur; une machine de guerre contre le colonialisme et le racisme, et c'est là sa justification. Mais ce n'est là qu'un aspect de la négritude, son aspect négatif.

Si nous avons tellement haï le colonialisme, si nous l'avons tellement combattu, c'est sans doute parce que nous avions conscience qu'il nous mutilait, qu'il nous humiliait, qu'il nous séparait de nous-mêmes et que cette séparation était intolérable : mais c'est aussi parce que nous savions qu'elle nous séparait du monde, qu'elle nous séparait de l'homme, de tous les hommes, y compris de l'homme blanc, bref qu'elle nous séparait de notre frère. Autrement dit, le poète de la négritude ne hait tellement le racisme et le colonialisme que parce qu'il a le sentiment que ce sont là des barrières qui empêchent la communication de s'établir.

Bref, si j'avais à définir l'attitude du poète de la négritude, la poésie de la négritude, je ne me laisserais pas désorienter par ses cris, ses revendications, ses malédictions. Ses cris, ses revendications, je ne les définirais que comme une postulation, irritée sans doute, une postulation impatiente, mais en tout cas une postulation de fraternité.

Et j'en arrive à l'objet même de ce colloque, le sens et la signification de l'art dans l'Afrique d'aujourd'hui.

On peut l'affirmer sans crainte, jamais l'Afrique n'a eu autant besoin de l'art. Jamais elle n'a eu autant besoin de son art, de son propre art. Cela est vrai bien sûr pour les raisons générales que j'évoquais tout à l'heure et qui sont valables pour le monde entier. Mais il s'ajoute à cela des raisons qui sont particulières à l'Afrique.

Quel est le grand phénomène des temps modernes? C'est que l'Afrique est entrée définitivement et tout entière dans l'aura et la mouvance de la civilisation européenne. Il suffit de dire cela pour que l'on comprenne à quel point l'Afrique est menacée. Menacée à cause de l'impact de la civilisation industrielle. Menacée par le dynamisme interne de l'Europe et de l'Amérique. On me dira : pourquoi parler de menace, puisqu'il n'y a pas de présence européenne en Afrique, puisque le colonialisme a disparu et que l'Afrique est indépendante?

Malheureusement, l'Afrique ne s'en tirera pas à si bon compte. Bien sûr, la colonisation, le colonialisme offraient le cadre rêvé pour que cet impact puisse agir dans les conditions 
d'efficacité optima. Mais ce n'est pas parce que le colonialisme a disparu que le danger de désintégration de la culture africaine a disparu. Le danger est là et tout y concourt, avec ou sans les Européens : le développement politique, la scolarisation plus poussée, l'enseignement, l'urbanisation, l'insertion du monde africain dans le réseau des relations mondiales, et j'en passe. Bref, au moment où l'Afrique naît véritablement au monde, elle risque comme jamais de mourir à elle-même. Cela signifie qu'il faut s'ouvrir au monde avec les yeux grands ouverts sur le péril et qu'en tout cas le bouclier d'une indépendance qui ne serait que politique, d'une indépendance politique qui ne serait pas assortie et complétée par une indépendance culturelle, serait en définitive le plus illusoire des boucliers et la plus fallacieuse des garanties.

L'histoire est toujours dangereuse. Le monde de l'histoire, c'est le monde du risque, mais c'est à nous qu'il appartient à chaque moment d'établir et de réajuster la hiérarchie des périls. Je dis qu'à l'heure actuelle le péril pour l'Afrique, ce n'est pas le refus du monde extérieur, ce n'est pas le refus d'ouverture, ce n'est pas le chauvinisme, ce n'est pas le racisme noir, c'est bien au contraire l'oubli d'elle-même, c'est l'acculturation et la dépersonnalisation.

Pour en revenir à mon propos du début, je dirai que le danger pour l'Afrique c'est d'entrer à son tour dans la réification. Et, cette fois, la réification ne jouera pas dans les relations avec l'autre. Dans le cas de l'Afrique, et c'est le comble du drame, la réification jouera dans les relations de l'Afrique avec elle-même. Si l'on n'y prend garde, l'Afrique risque de ne plus se voir que par les yeux des autres et de jeter sur elle-même un regard pétrifiant.

Je ne voudrais pas que l'on croie à une vue arbitraire. Je n'en veux pour preuve que la discussion qui s'est instaurée hier à la Commission des arts entre les éminents spécialistes venus d'Europe et d'Amérique : M. Goldwater, M. Laude, Michel Leiris. Au cours de ces discussions, M. Goldwater, parlant de l'influence de l'art africain sur l'art occidental, nous a dit qu'en réalité le mot «influence » était impropre, qu'il n'y a pas eu d'influence à proprement parler de l'art nègre sur les artistes européens et qu'il serait plus juste de dire qu'à un moment donné de l'histoire de l'art occidental l'art africain, rencontré par hasard, a servi de catalyseur à l'art occidental. Et cela est vrai. M. Laude a précisé et il a montré en particulier que Picasso ne s'est servi de l'art nègre que pour résoudre ses problèmes à lui, Picasso, et que si Picasso a contesté l'art occidental c'est à l'intérieur et non en dehors de l'art occidental. La question que je pose est celle-ci : est-ce que cela est vrai pour la majorité des artistes africains contemporains? Quand, éduqués par l'Europe et formés dans les écoles européennes, ils contestent, et c'est le droit, quand ils contestent l'art africain traditionnel, le contestentils à l'intérieur de l'africanité ou en dehors de l'africanité ? La réponse est malheureusement négative et $M$. Fagg a raison de dire que si l'art africain traditionnel a fini, a cessé d'être à l'heure actuelle le catalyseur de l'art occidental, il n'a pas encore commencé à être le catalyseur de l'art africain contemporain.

Voilà, n'est-il pas vrai, une remarque qui va loin et qui est significative des dangers que courent à l'heure actuelle l'homme d'Afrique, la culture de l'Afrique, l'art africain. M. Bastide l'a dit : ne viendra-t-il pas un moment où il n'y aura plus d'art africain et où il n'y aura plus qu'un art semblable à tous les autres arts du monde, avec cette seule différence - mais absolument secondaire, insignifiante, négligeable - qu'il aura été fait par des Africains et non par des Européens ou des Américains? Nul d'entre nous bien sûr ici n'est dans les secrets de l'histoire et nul ne peut donner de réponse à l'interrogation angoissée de $M$. Bastide.

Tout ce que nous pouvons dire, nous, hommes d'Afrique, nous, hommes de ce colloque, nous, hommes de culture, est que nous ne considérons pas comme souhaitable et comme un idéal à rechercher la substitution à l'art africain d'un art, les uns diront laudativement universel, les autres diront péjorativement cosmopolite, en tout cas non spécifique, fait par les Africains. 
Ici, j'entends l'objection d'André Malraux, qui nous dira et nous a dit : mille regrets, les souhaits et les vœux ne comptent pas en histoire. Il y a une évolution, une évolution qui est nécessaire. On nous a dit : essayons de retrouver l'âme africaine qui conçut les masques : à travers elle, nous atteindrons le peuple africain. Je n'en crois rien. C'est André Malraux qui parle : "Ce qui a fait jadis les masques comme ce qui a fait jadis les cathédrales est à jamais perdu. » Mais on peut répondre à André Malraux ceci : que le problème est mal posé et qu'il ne s'agit pas de refaire les masques, pas plus que pour l'Europe il ne peut s'agir de refaire les cathédrales.

Mais alors, me dira-t-on, que faut-il faire pour assurer à l'art africain - et non pas à l'art des Africains - une survie et une vitalité nouvelles dans un monde moderne pour lequel il n'a pas été fait et dont tous les éléments conspirent à sa disparition?

C'est là pour nous une question essentielle. Cette survie et cette vitalité nouvelles sontelles possibles ou sont-elles seulement envisageables? À cet égard, je suis, tout comme M. Bastide, beaucoup moins pessimiste qu'André Malraux. Plus exactement, je ne dirai pas que je suis optimiste, je dirai que la partie n'est pas jouée et qu'il dépend de nous, de nous tous, qu'elle soit gagnée.

Je crois que, lorsqu'on parle des chances de survie de l'art africain, l'erreur est de poser le problème en termes d'art. Ce n'est pas en termes d'art, c'est en termes humains qu'il faut poser le problème de l'art africain, et c'est la considération même du caractère spécifique de l'art africain qui nous mène à adopter cette optique. En effet, dans l'art africain, ce qui compte, ce n'est pas l'art, c'est d'abord l'artiste, donc l'homme. En Afrique, l'art n'a jamais été savoir-faire technique, car il n'a jamais été copie du réel, copie de l'objet ou copie de ce qu'il est convenu d'appeler le réel. Cela est vrai pour le meilleur de l'art européen moderne, mais cela a toujours été vrai pour l'art africain. Dans le cas africain, il s'agit pour l'homme de recomposer la nature selon un rythme profondément senti et vécu, pour lui imposer une valeur et une signification pour animer l'objet, le vivifier et en faire symbole et métalangage.

Autrement dit, l'art africain est d'abord dans le cœur et dans la tête et dans le ventre et dans le pouls de l'artiste africain. L'art africain n'est pas une manière de faire, c'est d'abord une manière d'être, une manière de plus être, comme dit le teilhardien Léopold Sédar Senghor.

Si cela est vrai, on comprend le double échec auquel nous assistons souvent : l'échec des artistes africains qui s'évertuent à copier des œuvres européennes ou à appliquer des canons européens. Mais aussi l'échec esthétique des artistes africains qui se mettent à copier du nègre en répétant mécaniquement des motifs ancestraux comme ces nègres bosches dont nous a parlé $M$. Bastide et qui, pendant un certain temps, pendant une certaine période de l'histoire, ont recopié, reproduit mécaniquement les modèles légués par leurs ancêtres ashantis. Il est clair que ces tentatives ne peuvent qu'échouer, car elles sont précisément à contre-sens de l'art africain. L'art africain n'est pas copie. Il n'est jamais copie, fût-ce de soimême, il n'est jamais reproduction, répétition, reduplication, mais au contraire inspiration, c'est-à-dire agression de l'objet, investissement de l'objet par l'homme qui a assez de force intérieure pour le transformer en une forme de totale communication (et non pas cette forme de communication appauvrie que constitue le langage).

L'art africain, comme tout grand art, me dira-t-on, en tout cas plus que tout autre, et depuis si longtemps si ce n'est depuis toujours, est d'abord dans l'homme, dans l'émotion de l'homme transmise aux choses par l'homme et sa société. C'est la raison pour laquelle on ne peut séparer le problème du sort de l'art africain du problème du sort de l'homme africain, c'est-à-dire en définitive du sort de l'Afrique elle-même.

L'art africain de demain vaudra ce que vaudront l'Afrique de demain et l'Africain de demain. Si l'homme africain s'appauvrit, s'il s'étiole, s'il se coupe de ses racines, s'il se prive 
- -

1. Source : Aimé Césaire, pour regarder le siècle en face, sous la direction de A. Thebia-Melsan, Paris, Maisonneuve et Larose, 2000 : 20-26. de ses sucs nourriciers, s'il se coupe de ses réserves millénaires, s'il devient le voyageur sans bagage, s'il se déleste de son passé pour entrer plus allégrement dans l'ère de la civilisation de masse, s'il se débarrasse de ses légendes, de sa sagesse, de sa culture propre, ou bien tout simplement s'il considère qu'il n'a plus aucun message à délivrer au monde, s'il a perdu son assurance historique ou s'il ne la retrouve pas, rien n'y fera malgré les festivals, malgré les encouragements officiels, malgré l'Unesco, malgré tous les prix, c'est très simple, l'art africain s'étiolera, s'appauvrira et disparaîtra.

$\mathrm{Si}$, au contraire, l'homme africain conserve et préserve sa vitalité, son assurance, sa générosité, son humour, son rire, sa danse, s'il se campe fièrement sur sa terre non pas pour s'isoler ou pour bouder, mais au contraire pour accueillir le monde, alors l'art africain continuera.

Bien sûr, il aura évolué, et mieux. Il se sera transformé, mais c'est tant mieux, comme se transforme d'époque en époque le contenu des rêves et de l'imagination de l'humanité. Mais cette évolution même et cette mutation seront le signe que l'art africain sera vivant et bien vivant. Aussi bien est-ce en nos mains, en nos mains à tous et non pas seulement entre les mains des hommes de culture, car la séparation est absolument artificielle, c'est entre nos mains à tous que se trouve l'avenir de l'art africain. C'est pourquoi, aux hommes d'État africains qui nous disent: Messieurs les artistes africains, travaillez à sauver l'art africain, nous répondons : Hommes d'Afrique et vous d'abord, politiques africains, parce que c'est vous qui êtes les plus responsables, faites-nous de la bonne politique africaine, faites-nous une bonne Afrique, faites-nous une Afrique où il y a encore des raisons d'espérer, des moyens de s'accomplir, des raisons d'être fiers, refaites à l'Afrique une dignité et une santé, et l'art africain sera sauvé ${ }^{1}$. 


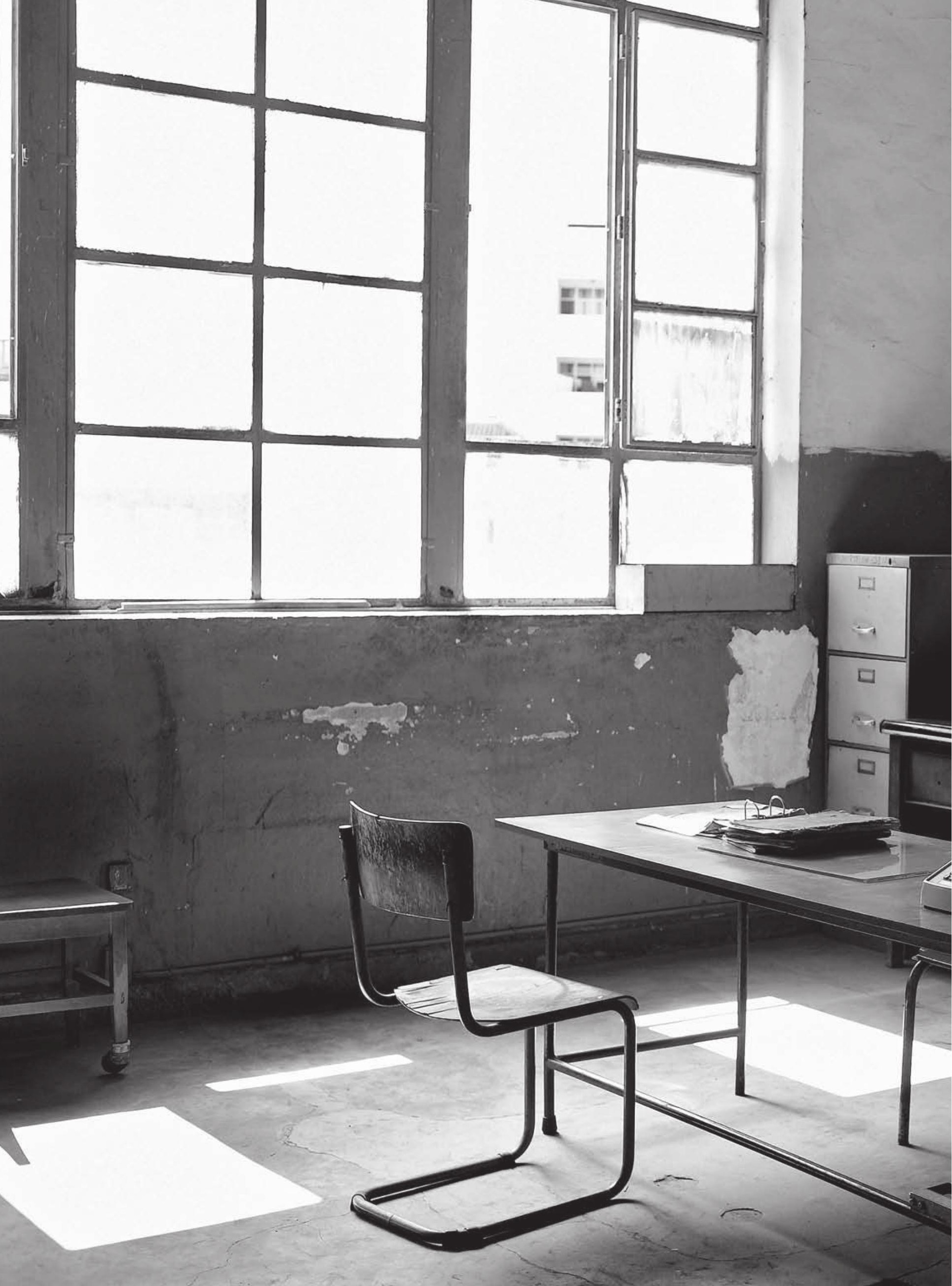




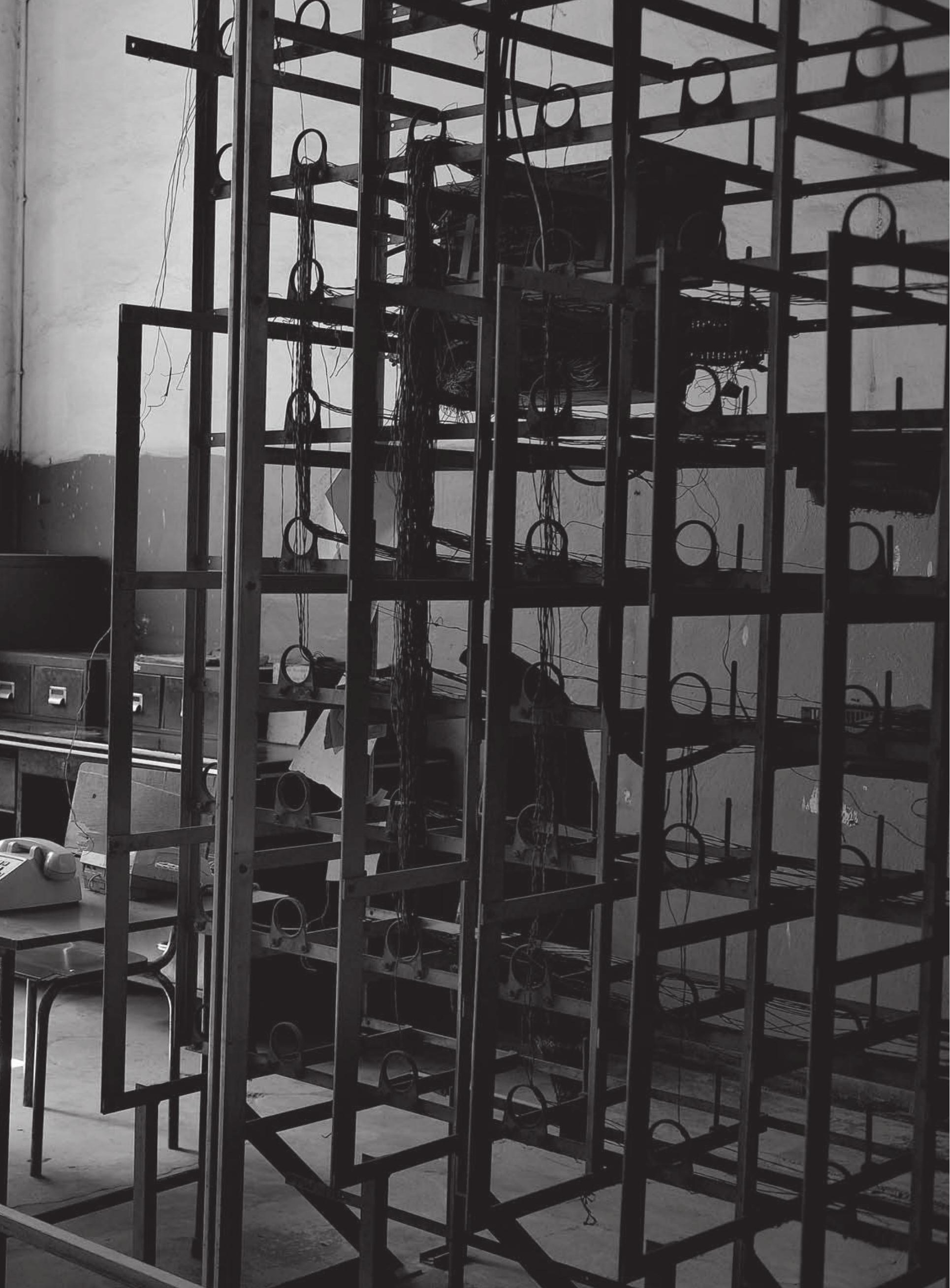

